\title{
PUFA: Consechencia DE LA CARIES NO TRATADA EN NIÑOS DE 6 A 12 AÑOS DEL CANTÓN AZOGUES
}

PUFA: CONSEQUENCE OF NOT TREATED CARIES IN CHILDREN FROM 6 TO 12 YEARS FROM AZOGUES COUNTY

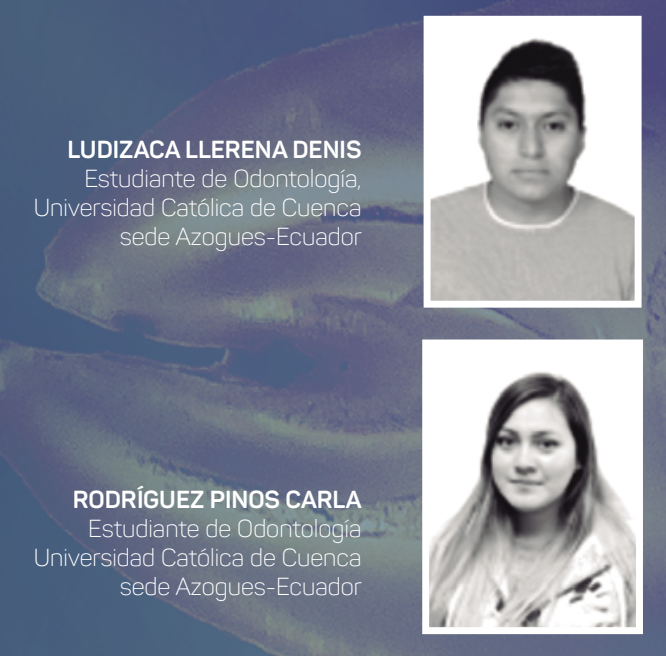

RESUMEN

La Organización Mundial de la Salud (OMS) en su último estudio de Salud Bucodental en América Latina, estima que aproximadamente el $60 \%$ a $90 \%$ de los escolares presenta caries dental, patología que desencadena lesiones pulpoperiapicales. Objetivo: Determinar la lesión pulpar más frecuente según el índice PUFA en niños de 6 a 12 años como consecuencia de caries dental no tratada. Métodos: El presente estudios es de tipo observacional, descriptivo y transversal, conto con una muestra total de 189 escolares. Se realizó un examen clínico considerando los criterios establecidos según el índice de PUFA.
DDS. SÁNCHEZ ORDOÑEZ MARÍA JOSÉ Especialista en Endodoncia e Implantologia Oral - Universidad de Buenos Aires y Católica Argentina Docente Catedra Endodoncia Carrera de Odontología de la Universidac

Católica de Cuenca sede Azogues.

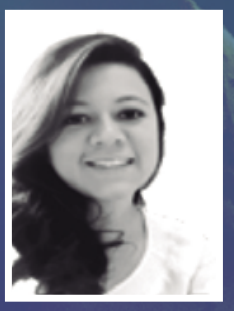

DDS. MONTESINOS RIVERA VANESSA Especialista en Endodoncia Universidad de Cuenca Docente Catedra Endodoncia Carrera de Odontología de la Universidad Católica de Cuenca sede Azogues-Ecuador

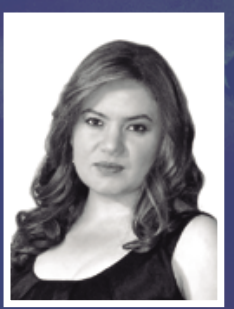

Autor para Correspondencia Vanessa Montesinos e-mail:. mmontesinosr@ucacue.edu.ec Fecha de Recepción: 15 de agosto de 2019 Fecha de Aceptación: 30 de septiembre de 2019

Página 25 a 30

Los datos obtenidos fueron analizados mediante el programa estadístico SPSS 23 (Statistical Package forthe Social Sciences) para establecer la frecuencia. Resultados: Del total de la población analizada predominaron los pacientes sanos (70,4\%), encontrando un porcentaje bajo de pacientes afectados (29,6\%). De las patologías que abarcan el índice PUFA en dentición decidua o permanente, la pulpitis fue la de mayor prevalencia (74,7\%), especialmente en el sexo femenino (54,65\%), afectando principalmente al grupo molar tanto en la dentición primaria como permanente. Conclusiones: La lesión pulpar más frecuente como consecuencia de caries dental no tratada en niños de 6 a 12 años es la pulpitis.

PALABRAS CLAVE: Caries Dental, Pulpitis, Inflamación Endodóntica, Enfermedad estomatognática. 


\section{SUMMARY}

The World Health Organization (WHO) in its latest study of Oral Health in Latin America, estimates that approximately 60\% to $90 \%$ of schoolchildren have dental caries, pathology that triggers octopus-periapical lesions. Objective: To determine the most frequent pulp lesion according to the PUFA index in children aged 6 to 12 years as a result of untreated tooth decay. Methods: An observational, descriptive and cross-sectional study was conducted, the total sample of the study was made up of 189 schoolchildren. A clinical examination was performed considering the criteria established according to the PUFA index. The data obtained were analyzed using the statistical program SPSS 23 (Statistical Package for the Social Sciences) to establish the frequency. Results: Healthy patients (70.4\%) predominated from the total population analyzed, finding a low percentage of affected patients (29.6\%). Of the pathologies that cover the PUFA index in deciduous or permanent dentition, pulpitis was the most prevalent (74.7\%), especially in females (54.65\%), mainly affecting the molar group in both primary and permanent dentitions. Conclusions: The most frequent pulp lesion as a result of untreated tooth decay in children 6 to 12 years is pulpitis.

KEYWORDS: Dental Decay,Pulpitis, Inflammation Endodontic, Stomatognathic Disease

\section{INTRODUCCIÓN}

La Organización Mundial de la Salud(OMS) define a la salud bucodental como «un estado exento de dolor bucodental o facial crónico, cáncer de la cavidad bucal o la garganta, infección oral y anginas, periodontopatías, caries dental, pérdida de dientes y otras enfermedades y trastornos que limitan la capacidad de una persona para morder, masticar, sonreír y hablar, así como su bienestar psicosocial»; en su último informe establece que más del 50\% de la población presenta enfermedades bucodentales como la caries dental, considerada la patología de mayor prevalencia, especialmente en la población infantil; Ecuador no es la excepción, el Ministerio de Salud Pública (MSP) establece que la caries dental, gingivitis, periodontopatías y la fluorosis dental destacan como las enfermedades más prevalentes en nuestro medio.(1) La caries dental esdefinida como una patología multifactorial, localizada y destructiva, causa la pérdida de la estructura dental debido a la acción microbiana que incluye fenómenos de desmineralización y re mineralización formando una lesión cavitaria que al no serdiagnosticada y tratada de manera temprana origina infecciones orales, necrosis pulpar hasta una posible pérdida de la estructura dental, desencadenando problemas funcionales, estéticos y sistémicos, afectando el bienestar psico-social de los individuos. (2-4)

El índice CPOD/ceod han sido empleado para determinar la presencia de caries y la terapéutica quirúrgica y rehabilitadora sin considerar las patologías pulpares y periapicales subsecuente a una lesión cariosa no tratada motivo por el que, el índice de PUFA/pufa permite obtener de manera certera datos estadísticos en poblaciones de bajos recursos cuyos índices de caries dental son elevados requiriendo atención temprana y oportuna, creado por Monse et al. éste índice registra afecciones orales como consecuencia clínica de una lesión cariosa no tratada, reconociendo solamente las piezas dentales que presenten afección pulpar visible por caries dental. Presenta cuatro códigos y criterios identificados en letras mayúsculas para la dentición permanente y en minúsculas para la dentición temporal que involucra la presencia de una pulpitis, úlcera, fístula o absceso con las siguientes consideraciones: (5-9)

P: Significa una pulpa involucrada, ejemplo: diente con una cámara pulpar abierta o con una destrucción extensa de la parte coronal con raíces restantes.

U: Significa una ulceración de la mucosa oral debido a bordes afiladosde un diente con afectación pulpar.

F: Es para una fístula activa

A: Es para un absceso" (10)

La puntuación PUFA/pufa se calcula de manera individual, en base al índice CPOD o ceod, se registran el número de dientes que cumplen con los criterios antes mencionados. Para una dentición temporal la puntuación será de 0 a 20 y para la permanente 0 a 32. La prevalencia se calcula con el porcentaje de la población o una puntuación de uno o más. Se puede realizar con o sin la ayuda de un instrumento, para calcular el rango de afección por persona, aplicando la siguiente formula: (5)

$$
\frac{(P \cup F A+p u f a)}{(D+d)} * 100
$$

Diversas campañas de salud oral e higiene bucal han sido desarrolladas a lo largo de las últimas décadas alrededor del mundo a pesar de ello, la caries no deja de ser el principal problema de salud pública por el que se acude a consulta. Factores económicos, nivel de educación, lugar de residencia y hábitos 
del paciente son considerados factores directos en el desarrollo de las patologías buco-dentales que influyen en el avance de las lesiones; la caries dental no tratada a tiempo puede desencadenar diferentes patologías, que resultan de la falta de un tratamiento temprano, originando complicaciones que ocasionan emergencias en niños y adultos. (6) Estudios previos han determinado la necesidad de tratamiento endodóntico debido a la existencia de lesiones cariosas no tratadas mediante el índice de PUFA/pufa, investigaciones como el de Vélez et al. (11) realizados en Cuenca-Ecuador determinaron que la incidencia de pulpitis en escolares fue del 9.7\% siendo el primer molar superior la pieza dental con mayor compromiso pulpar (61.1\%) mientras que en las parroquias urbanas Gil Ramírez, Bellavista y San Sebastián, Cuenca-Ecuador el promedio de afectación pulpar fue del $6 \%$ sin valores significativos de fistulas, ulceras y abscesos. (12)En la parroquia "Hermano Miguel" Cuenca -Ecuador se evidenció un índice bajo en el sexo femenino 1.7\% y masculino 2.3\% (13)

En la ciudad de Azogues-Ecuador,no existen datos que determinen la condición pulpar,por lo tanto, el objetivo de la presente investigación fue determinar la lesión pulpar más frecuente según el índice PUFA en niños de 6 a 12 años como consecuencia de caries dental no tratadaconsiderando el sexo, la edad y la pieza dental másafectada.

\section{MATERIALES Y MÉTODOS}

Se realizó un estudio de tipo observacional, descriptivo y transversal. La población total fue de 375 niños, matriculados en la Unidad Educativa Particular Universitaria de Azogues y Unidad Educativa Guapán, se aplicaron como criterios de inclusión:

\section{· $\quad$ Escolares de 6 a 12 años. \\ - Consentimiento y asentimiento infor- mado autorizados.}

La muestra total de estudio la conformaron 189 escolares. Previa aprobación por parte delos rectores y de los padres de familia se inició con la toma de datos con dos per- sonas capacitadas y calibradas, realizando un examen clínico a todos los niños con la ayuda de un set básico de diagnóstico. La evaluación de los puntajes de pufa se basósolo en el examen visual sin instrumentación. (10)Los datos recolectados en una ficha de diagnóstico, fueron almacenados en una base de datos en Microsoft Excel, siendo analizados en el programa estadístico SPSS 23 (StatisticalPackageforthe Social Sciences) obteniendo tablas de frecuencias y porcentajes. Esta investigación no implico aspectos bioéticos.

\section{RESULTADOS}

La muestra estuvo constituida por 91 pacientes del sexo masculino (48,1\%) y 98 del sexo femenino(51,9\%), la edad de los individuos estuvo comprendida entre 6 y 12 años, predominando el grupo de edad de 6 a 9 años (58,8\%). (Tabla 1).

Del total de la población analizada prevalecieron los pacientes sanos (70,4\%), el resto de individuos presentaron una o varias de las patologías que comprende el índice PUFA en dentición decidua o permanente. Existió un predominio de Pulpitis (74,7\%), seguida de Ulceras (20\%) mientras que Fistulas y Abscesos se presentaron en iguales proporciones (2,6\%), siendo las menos frecuentes. La Pulpitis fue encontrada mayoritariamente en la dentición decidua en contraste con Ulceras, Fistulas y Abscesos que fueron halladas con más frecuencia en dentición permanente. (Tabla 2)

La patología más prevalente en el sexo femenino fue la pulpitis en ambas denticiones mientras que en el sexo masculino destacan con un 26,08\% la presencia de ulceras en la dentición permanente seguido de pulpitis en la dentición decidua (22,09\%). (Tabla 3)

Se examinaron 116 piezas dentales afectadas, el 70,7\% corresponde a la dentición decidua donde el primer y segundo molar fueron afectados por igual (46,3\%), en cuanto al grupo permanente, el primer molar (52,9\%) fue la pieza dominante, mientras que en el grupo incisivo no se encontraron casos. (Tabla 4)

\section{DISCUSIÓN}

El presente estudio fue realizado para determinar la necesidad de tratamiento endodóntico mediante un examen clínico a niños de 6 y 12 años que se encuentran en el proceso de cambio dentario mediante el índice de PUFA/pufa. La mayoría de la población examinada no mostró afección 70,4\% frente a un 29,6\%. La prevalencia del índice PUFA/pufa fue del 2.63\%, estos valores son similares a otro estudio con un índice de 1.29\% (14)sin embargo, otras investigaciones destacan valores mayores 56\% (5),37\% (15) y 15.9\% (16).La consecuencia de caries no tratada más prevalente fue la pulpitis $74,7 \%$ con un 88,4\% en la dentición temporal. Estos resultados coinciden con estudios previos (17-19), quienes establecieron que la pulpitis es la lesión con mayor predominio 
en un21.2\%,13\% y 10.73\% respectivamente, estos resultados pueden deberse a que,en la dentición decidua, las terminaciones nerviosas se encuentran en menor proporción cuando la pieza dental esta próxima a su exfoliación, y el efecto sensorial inflamatorio es menor, en comparación con la dentición permanente por lo que un niño puede desarrollar una patología pulpar de forma asintomática. (20)

La úlcera destaco como la segunda patología más prevalente (20.0\%), concordando con investigaciones previas que reportan porcentajes del 36.7\% y del 15\% respectivamente. (8) (21) sin embargo otros estudios identificana la fístula (0.6\%), como la patología que le sigue a la pulpitis(19).

En investigaciones previas el sexo femenino presentó mayor prevalencia,(22-23)en el presente estudio la afección fue del (51.9\%). En relación a la edad, se encontró que el menos afectado por la lesiones pulpares fue el de 10 a 12 años con el 41.2\%, estos resultados pueden estar relacionados con elproceso de cambio dentario el cual culmina aproximadamente a los 13 años con la erupción de los segundos molares superiores y que oscila entre los seis y ocho años en donde coexisten piezas dentales de diferentes denticiones cuyo tiempo de erupción está relacionado con factores locales, hereditarios, raza, estados nutricionales y patológicos entre otros, pudiendo modificar este proceso. (24) La muestra de estudio presento una dentición mixta estableciendo que el grupo dentario más afectado fueron los primeros molares permanentes en un 52.9\% al igual que en la dentición decidua con un 46.3\%, encontrándose similitud con lo establecido en otro estudio (25), donde los primeros molares superiores permanentes son los más perjudicados (4\%), lo que puede deberse a que la pulpa en dientes jóvenes de piezas permanentes es más grande e inmadura presentando mayor susceptibilidad a la acción bacteriana,(26)lo que determina la necesidad de reforzar las campañas preventivas y de intervención que permitan tratar de manera temprana éstas piezas dentales debido a su importancia y a la funcionalidad que cumplen dentro del arco dentario y en el desarrollo maxilo facial de los niños.

Dentro de las limitaciones se destaca la falta de colaboración de algunos padres de familia motivo por el que, se recomienda establecer campañas que sean dirigidas a ellos o a sus representantes debido a que, la falta de conocimiento o temor impide que exista acercamiento por parte de las entidades de Educación Superior a sectores en donde se requiererealizar un levantamiento estadístico de las condiciones buco dentales de la población que permitan desarrollar programas educativos y preventivos, que mejoren las condiciones bucodentales de la población debido a que, la caries dental puede tener repercusiones significativas en la calidad de vida de los niños requiriendo un diagnóstico temprano. Si bien el índice de PUFA/pufa fue bajo, la presente investigación es un precedente para realizar futuras investigaciones que permitirá un mayor involucramiento por parte de las entidades públicas y privadas en la provincia del Cañar.

\section{CONCLUSIONES}

Las afecciones pulpo-periapicales como consecuencia de una caries no tratada fue la pulpitis (74.7\%), con un mayor porcentaje en el sexo femenino, siendo el primer molar la pieza dental más afectada (46.3\%) en ambas denticiones

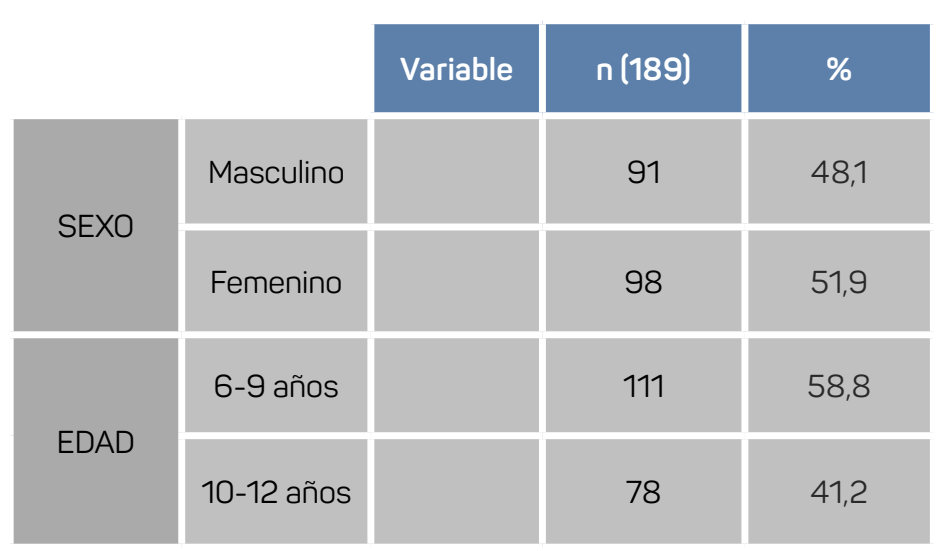

Tabla 1

Distribución de la muestra de escolares de 6-12 años 


\begin{tabular}{|l|c|c|}
\hline \multicolumn{1}{|c|}{ Variable } & Frecuencia & $\%$ \\
\hline PUFA & & \\
\hline Enfermos & 56 & 29,6 \\
\hline Sanos & 133 & 70,4 \\
\hline Pulpitis & 86 & $74,7^{*}$ \\
\hline Dentición permanente & 10 & 11,6 \\
\hline Dentición decidua & 76 & $88,4^{*}$ \\
\hline Ulceras & 23 & 20 \\
\hline Dentición permanente & 20 & 87 \\
\hline Dentición decidua & 3 & 13 \\
\hline Fístulas & 3 & 2,6 \\
\hline Dentición permanente & 2 & 66,7 \\
\hline Dentición decidua & 1 & 33,3 \\
\hline Abscesos & 3,6 \\
\hline Dentición permanente & & 33,3 \\
\hline Dentición decidua & & 2 \\
\hline
\end{tabular}

\begin{tabular}{|c|c|c|c|c|}
\hline Sexo & \multicolumn{2}{|c|}{ Frecuencia } & \multicolumn{2}{c|}{ Porcentaje } \\
\hline Femenino & Permanente & Deciduo & Permanente & Deciduo \\
\hline Pulpitis & $17^{*}$ & $47^{*}$ & 19,76 & 54,65 \\
\hline Ulceras & 14 & 3 & 60,86 & 13,64 \\
\hline Fistulas & 1 & 0 & 33,3 & 0 \\
\hline Abscesos & 1 & 1 & 33,3 & 33,3 \\
\hline Masculino & Permanente & Deciduo & Permanente & Deciduo \\
\hline Pulpitis & 3 & $19 *$ & 3,48 & 22,09 \\
\hline Ulceras & $6 *$ & 0 & 26,08 & 0 \\
\hline Fistulas & 1 & 1 & 33,3 & 33,3 \\
\hline Abscesos & 1 & 0 & 33,3 & 0 \\
\hline
\end{tabular}




\begin{tabular}{|l|c|c|}
\hline \multicolumn{1}{|c|}{ Variable } & Frecuencia & $\%$ \\
\hline Grupodentario & & \\
\hline Permanente & 34 & 29,3 \\
\hline Deciduo & 82 & 70,7 \\
\hline Grupo dentario & & \\
\hline permanente & & \\
\hline Incisivo Central & 0 & 0 \\
\hline Incisivo Lateral & 0 & 0 \\
\hline Canino & 2 & 5,9 \\
\hline Primer Premolar & 3 & 8,8 \\
\hline Segundo Premolar & 10 & 29,4 \\
\hline Primer Molar & $18^{*}$ & 52,9 \\
\hline Segundo Molar & 1 & 2,9 \\
\hline Dentición Decidua & & \\
\hline Incisivo Central & 0 & 0,3 \\
\hline Incisivo Lateral & 1 & 46,3 \\
\hline Canino & $58^{*}$ & \\
\hline Primer Molar & & \\
\hline Segundo Molar & & \\
\hline
\end{tabular}

Tabla 4.

Análisis del grupo Dentario

\section{REFERENCIAS BIBLIOGRÁFICAS}

1. Cabeza G, González F, Paredes Cl. Estado de Salud Oral en el Ecuador. Articulo Opinión. Revista OACTIVA UC Cuenca. 2016;1(3):65-70.

2. Velásquez V. Reacción de la pulpa dentaria ante la presencia de la caries y los tratamientos dentales. Odontol. Sanmarquina 2010; 13(1):28-31

3. Núñez D, García L. Bioquímica de la caries dental. Revista Habanera de Ciencias Médicas 2010:9(2) 156-166

4. Villena R, Pachas Fl: Sánchez Yh; Carrasco M. Prevalencia de caries de infancia temprana en niños menores de 6 años de edad, residentes en poblados urbano marginales de Lima Norte. Revista Estomatológica Herediana,2011: 21 (2):79-86 5. Monse B, WeltzienR,Benzian H, Holgren C, Van Palenstein, Heldeman W, PUFA An index of clinical consequences of untreated dental caries Community Dent Oral Epidemiol 2010; 38:77-82

6. Rivera L, Rodríguez M. Necesidad de Tratamiento Endodóntico en Escolares de 6 años de Edad de Dos Parroquias de Cuencadissertation.Cuenca: Universidad Católica de Cuenca; 2016:120.

7. Bhoopathi P, Prathibha B, Parthasarthi R, Monica M, Rajesh R. Co-relation between PUFA Index and Oral Health Related Quality of Life of a Rural Population in India: A Cross-Sectional Study. Journal of Clinical and Diagnostic Research. 2015: 9 (1) 39-42

8. Graciano L, Lesiones Cariosas No Tratadas Según el Indice P.U.F. A en Escolares de Primera y Segundo Grado de Primaria de la Institución Educativa Cesar Vallejo, Distrito de Yauya Ancash dissertation.Lima-Perú:UniversidadWeiner; 2016; 66. 9. Carrasco M, Orejuela F, Consecuencias clínicas de caries dental no tratada en preescolares y escolares de instituciones educativas públicas; RevEstomatol Herediana. 2018; 28(4): 223-228

10. Bagińska J1, , Rodakowska E1, Wilczyńska-Borawska M1, Jamiołkowski $\mathrm{J} 2$, Index of clinical consequences of untreated dental caries (pufa) in primary dentition of children from north-east Poland, Rev. • Advances in Medical Sciences, 2013: 58 (2) $442-447$

11. Vélez E. Prevalencia de las consecuencias de caries dental no tratada en escolares de 12 años en la parroquia el Vecino - Cuenca. RevOactiv UC Cuenca. 2016;3(2):10-5

12. Poma SF, Lara MA. Necesidad de tratamiento endodóntico en escolares de 12 años de parroquias de Cuenca, 2016 - 2017. Tesis. Cuenca: Universidad Católica de Cuenca; 2017

13. Cárdenas J. Prevalencia de las enfermedades pulpares en piezas dentales con caries no tratadas mediante el índice de PUFA en escolares de 12 años de la parroquia "Hermano Miguel". Tesis. Cuenca: Universidad Católica de Cuenca; 2016. 14. Pachas F. García C. Carrasco M. Manrique J. Orejuela F. Córdova D. Castillo D. Villavicencio E. Mellado J. Machicado E. Diagnóstico epidemiológico sobre caries dental y consecuencias clínicas en escolares de Ventanilla. Rev. de la Facultad de Estomatología Master Challange.2014.

15. Shanbhog R y col. Clinical Consequences of Untreated Dental Caries Evaluated Using PUFA Index in Orphanage Children from India. 2013; 5 (5): 1-9.
16. Ramsha K. Warda F. Mehreen R. Faisal J. Clinica consequences of untreated dental caries assessed using PUFA index and its covariates in children residing in orphanages of Pakistan. Rev Kamran et al. BMC Oral Health. 2017; 17(108): 2-7

17. Astudillo A, Encalada L. Índice P.U.F.A en escolares de 12 años Cuenca-Ecuador [dissertation]. Cuenca: Universidad de Cuenca; 2017. 73p.

18. Aquino C. Pariona M. Consecuencias clínicas de caries dentales no tratadas en escolares de zonas rurales en Perú. Rev. OACTIVA UC Cuenca. 2017; 2(1):1-6.

19. Baginska J. Stokowska W. Pulpal InvolvementRoots-Sepsis Index: A New Method for Describing the Clinical Consequences of Untreated Dental Caries. RevMedPrincPract 2013; 22.555-560

20. Betancourt F, Fernández M. Valcarcel J.

Lesionespulpares y periapicalesenescolares del área de atención del PoliclínicoDocente de Playa. ciudad de la Habana. Rev HabanCiencMéd. 2009; 8 (2):1-8

21. Loyola R. Prevalencia de Caries no Tratadas Mediante Índice PUFA en Escolares de 12 Años de la Parroquia "EL Sagrario" en la Ciudad de Cuenca provincia del Azuay-Ecuador [dissertation]. Cuenca: Universidad Católica de Cuenca; 2016; 53. 22. Quizhpi G, Cuenca K, Zhunio K, Domínguez C. Prevalencia de Enfermedades Pulpares en Piezas con Caries no Tratadas en Escolares de la Parroquia "El Vecino" en la Ciudad de Cuenca Provincia del AzuayEcuador. 2017; 4(1).; 46-54

23. Rivera D, Bastidas M. Necesidad de tratamiento endodóntico en escolares de seis años en dos parroquias de la ciudad de Cuenca - 2016. Revista OACTIVA UC Cuenca. 2016; 1(3): 15-18.

24. Alzaete F, Serrano L, Cortes L, Torres E, Rodríguez M, Cronología y secuencia de erupción en el primer periodo transicional. Ces Odontología, 2016; 57 - 69 25. Palacios S, Bastidas M, Montesinos M, Aguilar J. Consecuencia de caries no tratada, en escolares de 12 años de edad en Cañaribamba Ecuador. Rev. Postgrado Scientiarvm. 2018; 4 (2) 43-46

26. Mendiburu C, Peñaloza R, Chuc I, Medina S. Enfermedades pulpares y periapicales en estructuras dentales permanentes en pacientes con edades de seis-catorce años. Revista Cubana de Estomatología 2017;54(3): 1-10. 